\title{
Consideraciones en pacientes con dolor crónico sometidos a cirugía
}

\author{
Catalina Kychenthal L. ${ }^{1}$, Eduardo Vega P. ${ }^{2}$ y Francisca Elgueta LB. ${ }^{2}$
}

\section{Considerations in chronic pain patients that will undergo surgery}

Chronic pain is an increasingly prevalent condition, estimated in approximately $32 \%$ of the Chilean population. These patients have characteristics that make optimal perioperative care more complex, especially in terms of analgesia, adverse effects of the different drugs, and post-surgical complications. The aim of this publication is to expose the growing problem involved in the management of patients with chronic pain undergoing surgery, and to highlight some considerations that must be taken. Among the most relevant aspects are an adequate characterization of pain and a good analgesic scheme to be used in the perioperative period, preferring a multimodal analgesia approach adjusted according to the patient's needs. Special consideration should be given to patients with chronic opioids use, those with neuropathic pain and those with fibromyalgia. An adequate follow-up of these groups will allow adjusting the schemes according to their needs and achieve a better perioperative analgesic management.

Key words: chronic pain; perioperative period; perioperative care; postoperative pain.

\section{Resumen}

El dolor crónico es una condición cada vez más prevalente, que afecta aproximadamente a un $32 \%$ de la población nacional. Estos pacientes poseen características particulares que complejizan un óptimo cuidado perioperatorio, sobre todo en términos de analgesia, efectos adversos de los distintos fármacos y complicaciones postquirúrgicas. El objetivo de esta revisión es exponer el creciente problema que involucra el manejo de los pacientes con dolor crónico no oncológico sometidos a una cirugía, y destacar algunas consideraciones que se deben tener en su cuidado. Dentro de los aspectos más relevantes están una adecuada caracterización del dolor y un buen esquema analgésico a ser utilizado en el perioperatorio, prefiriendo un enfoque multimodal ajustado acorde a las necesidades del paciente. Se debe tener especial consideración en pacientes usuarios crónicos de opioides, aquellos con dolor neuropático y aquellos con fibromialgia. Un adecuado seguimiento de estos grupos permitirá ajustar los esquemas de acuerdo con sus necesidades y lograr un mejor manejo analgésico perioperatorio.

Palabras clave: dolor crónico; periodo perioperatorio; cuidado perioperatorio; dolor postoperatorio.

\section{Introducción}

El dolor crónico no oncológico (DCNO) es un problema altamente prevalente en nuestro país, afectando a un $32,1 \%$ de la población ${ }^{1}$. Esto tiene implicancias en la condición basal de los pacientes, morbilidad, complicaciones postoperatorias y una mayor dificultad en el manejo perioperatorio ${ }^{2}$. En general, los pacientes usuarios crónicos de opioides desarrollan diversos efectos secundarios a su consumo, dificultando el manejo integral de estos pacientes. Estos incluyen la tolerancia (diminución de la respuesta de forma progresiva, obligándonos a aumentar las dosis), la dependencia (impidiéndonos la suspensión brusca) y la hiperalgesia (donde el aumento de dosis de opioides genera un aumento en el dolor $)^{2}$. Además, en los pacientes con DCNO, la mayor prescripción de opioides se ha asociado a un aumento dosis-dependiente de complicaciones postoperatorias, principalmente tromboembólicas, gastrointestinales e infecciosas ${ }^{3}$.

En este escenario clínico, estos pacientes son un desafío para el manejo conjunto que realizan los cirujanos y anestesiólogos. Existe, además, poca evidencia de buena calidad con respecto al tema, siendo históricamente el DCNO un criterio de exclusión en
Facultad de Medicina, Pontificia Universidad Católica de Chile. Santiago, Chile. 2División de Anestesiología, Facultad de Medicina Pontificia Universidad Católica de Chile. Santiago, Chile.

Recibido el 27 de julio de 2020 y aceptado para publicación el 21 de agosto de 2020 .

Correspondencia a: Dra. Francisca Elgueta LB elguetafrancisca@yahoo.com 
grandes estudios. El objetivo de esta revisión es exponer el creciente problema que implica el manejo de los pacientes con DCNO sometidos a una cirugía y enfatizar las principales consideraciones que se deben tener, específicamente en el contexto local. Se entregan, además, sugerencias y consideraciones prácticas para el manejo perioperatorio de estos pacientes en las distintas etapas de su atención.

\section{Consideraciones preoperatorias}

El primer acercamiento a los pacientes con DCNO que serán sometidos a una cirugía, generalmente se realiza de forma ambulatoria en las consultas previas a la intervención. Reconocer la existencia del dolor crónico en estos pacientes suele ser un primer paso necesario para obtener su confianza $\mathrm{y}$, por consiguiente, una buena anamnesis. Aquí es fundamental realizar una buena caracterización del dolor, incluyendo su intensidad (0 a 10 , siendo cero la ausencia de dolor y diez el peor dolor imaginable), localización, irradiación, tipo de sensación dolorosa (ej. choque eléctrico, punzante, cólico, etc.), factores agravantes/atenuantes, tiempo de evolución y el impacto en la calidad de vida. Debe ponerse especial énfasis en la totalidad de los medicamentos utilizados por el paciente, incluyendo sus dosis, efectos adversos, frecuencia, tiempo desde el inicio de su consumo y porcentaje de alivio del dolor con la terapia actual.

En usuarios crónicos de opioides, debido al aumento en el riesgo de complicaciones perioperatorias, deben buscarse comorbilidades dirigidamente. Estas deben ser estudiadas mediante la anamnesis, examen físico y exámenes de laboratorio o imágenes, buscando sobre todo factores de riesgo cardiovascular, gastrointestinales o de enfermedades infecciosas ${ }^{4}$.

Posterior a la evaluación inicial y previo a la cirugía, debe planificarse el plan analgésico que se utilizará, prefiriendo el uso de analgesia multimodal y multidisciplinaria. El concepto de analgesia multimodal implica el uso de analgésicos, que actúan a través de diversos mecanismos de acción con el fin de generar sinergia farmacológica y maximizar sus efectos beneficiosos, al mismo tiempo que se minimizan sus efectos colaterales ${ }^{5}$. En la Tabla 1 se

Tabla 1. Fármacos y dosis utilizadas en esquemas de analgesia multimodal

\begin{tabular}{|c|c|}
\hline Fármaco/técnica & Dosis \\
\hline Anestesia regional & $\begin{array}{l}\text { Siempre considerar anestesia regional (bloqueo de nervio periférico, peridural, bloqueos fasciales o infiltración de herida } \\
\text { operatoria) } \\
\text { Lidocaína dosis máxima } 5 \mathrm{mg} / \mathrm{kg} \\
\text { Bupivacaína dosis máxima } 2 \mathrm{mg} / \mathrm{kg} \\
\text { En caso de sobrepasar dichas dosis o administración intravascular existe el riesgo de intoxicación por anestésicos locales }\end{array}$ \\
\hline Paracetamol & $\begin{array}{l}1 \mathrm{~g} \text { cada } 6-8 \mathrm{~h} \text { adultos sanos (máximo } 4 \mathrm{~g} \text { /día) vía oral o endovenosa } \\
1 \mathrm{~g} \text { cada } 8 \mathrm{~h} \text { ancianos (máximo } 2-3 \mathrm{~g} / \text { día) vía oral o endovenosa }\end{array}$ \\
\hline Antiinflamatorios & $\begin{array}{l}\text { Ketorolaco } 10 \mathrm{mg} \text { cada } 8 \text { h vía oral o } 30 \mathrm{mg} \text { cada } 8 \text { h vía endovenosa } \\
\text { Ketoprofeno } 50 \mathrm{mg} \text { cada } 6-8 \text { h vía oral o } 100 \mathrm{mg} \text { cada } 8 \text { h vía endovenosa } \\
\text { Celecoxib } 200 \text { cada } 12-24 \text { h vía oral } \\
\text { Parecoxib } 40 \mathrm{mg} \text { cada } 12-24 \text { h vía endovenosa } \\
\text { Considerar efecto techo, no mezclar antiinflamatorios }\end{array}$ \\
\hline Ketamina & $\begin{array}{l}\text { Bolo endovenoso de } 0,25-0,5 \mathrm{mg} / \mathrm{kg} \text { después de la inducción anestésica y luego adicionar infusión } 0,25-0,5 \mathrm{mg} / \mathrm{kg} / \mathrm{h} \\
\text { durante el intraoperatorio } \\
\text { Suspender entre } 30-60 \mathrm{~min} \text { previos al fin de la cirugía o mantener en el postoperatorio con dosis bajas }(0,1-0,2 \mathrm{mg} / \mathrm{kg} / \mathrm{h})\end{array}$ \\
\hline Magnesio & Dosis: $40-50 \mathrm{mg} / \mathrm{kg}$ endovenosos en $15 \mathrm{~min}$ \\
\hline Lidocaína & Bolo $1,5 \mathrm{mg} / \mathrm{kg} \rightarrow$ infusión $1,5 \mathrm{mg} / \mathrm{kg} / \mathrm{h}$ \\
\hline$\alpha 2$ agonistas & $\begin{array}{l}\text { Clonidina vía oral } 75-100 \mathrm{ug} \text { previo a cirugía } \\
\text { Dexmedetomidina } 0,3-0,8 \mathrm{ug} / \mathrm{kg} / \mathrm{h} \text { endovenosa (se requiere que el paciente esté monitorizado) }\end{array}$ \\
\hline
\end{tabular}


presentan algunos de los analgésicos más comúnmente empleados en el perioperatorio de pacientes con DCNO.

Al momento de elegir el esquema analgésico, debe tenerse en cuenta el lograr una analgesia efectiva al mismo tiempo en que se eviten interacciones farmacológicas y síntomas de deprivación para los pacientes en los que esto aplique. Debido a esto, la indicación debe ser ajustada según cada paciente.

Se deben mantener los fármacos opioides y no opioides que el paciente esté consumiendo de forma ambulatoria para su dolor y a este esquema añadir nuestra analgesia multimodal para el periodo postoperatorio. Este esquema puede contar con el uso de paracetamol o antiinflamatorios no esteroidales (AINEs), en caso de que el paciente no presente alguna contraindicación para su administración (enfermedad renal, alergia, alteración de la coagulación, úlcera péptica, embarazadas en el tercer trimestre o enfermedad inflamatoria intestinal). Según el tipo de dolor pueden agregarse fármacos adyuvantes, destacando el uso de gabapentinoides (gabapentina o pregabalina) en pacientes con dolor neuropático o fibromialgia. Sobre estos, no hay sustento en la literatura para iniciarlos previo a la cirugía en pacientes que no los utilizan ${ }^{6}$, pero debiesen mantenerse las dosis previas en caso de usuarios crónicos ${ }^{7}$. Para la optimización de la analgesia perioperatoria debiesen considerarse técnicas de anestesia regional (bloqueo epidural, bloqueo de nervio periférico, bloqueo de planos fasciales o infiltración de la herida operatoria), acompañadas o no de anestesia general durante la cirugía. En caso de pesquisar ansiedad importante, se puede utilizar un ansiolítico que ayude al paciente a calmar su angustia, generalmente una benzodiacepina ${ }^{8}$, aunque la pregabalina ha demostrado también ser muy útil en este escenario clínico9.

Para pacientes usuarios crónicos de opioides, deben tenerse en cuenta algunas consideraciones específicas en cuanto a su tratamiento. Los pacientes deben consumir su dosis habitual de opioides el día de la cirugía, ya que tanto los efectos analgésicos excesivos como insuficientes conllevan riesgos. Esto incluye a usuarios de parches de fentanilo o buprenorfina ${ }^{10}$, quienes no deben retirarlos sino colocarlos en un sitio que no interfiera con la cirugía. Debido al riesgo de prolongación del segmento QT y arritmias malignas, los pacientes que utilicen metadona deben tener un electrocardiograma previo a la cirugía, en especial aquellos que tengan factores de riesgo o consuman otros fármacos prolongadores del QT.

Deben ser derivados al especialista en dolor los casos de mayor complejidad, incluyendo a pacientes con dolor crónico tratados con polifarmacia, dolor crónico no controlado con tratamiento actual, usuarios crónicos de opioides fuertes (Tabla 2), patología psiquiátrica inestable, hospitalizaciones previas por dolor, pacientes en tratamiento actual por unidad multidisciplinaria de dolor y aquellos con historia de dificultad en el manejo de dolor postoperatorio en cirugía previa (Figura 1).

\section{Consideraciones postoperatorias}

En el período postoperatorio, los pacientes con dolor crónico suelen ser más complejos de manejar, e idealmente requieren de un enfoque multidisciplinario y multimodal (Figura 2). La analgesia controlada por el paciente (PCA por sus siglas en ingles) y técnicas de anestesia regional cobran gran importancia según tipo de cirugía, dolor postoperatorio esperado y el tipo de paciente con DCNO. El esquema analgésico postoperatorio debiese incluir paracetamol y AINEs de forma horaria, además, de los fármacos opioides según el caso y siempre adicionar una alternativa de rescate (no prescribir un AINEs en caso de que el paciente ya tenga uno de forma horaria). Del mismo modo que fue mencionado previamente, existen fármacos adyuvantes como los gabapentinoides o antidepresivos tricíclicos para el dolor neuropático o fibromialgia e infusiones de drogas anestésicas (lidocaína y/o ketamina) que deben manejarse en conjunto con el equipo de anestesiología $^{11,12}$.

Estudios describen que los pacientes con DCNO usuarios de opioides requieren, en promedio, tres a cuatro veces la cantidad de dosis de opioides postoperatorios en comparación con aquellos que no lo son $^{4,13}$. En pacientes con dolor oncológico, esta cifra se mantiene y la duración del tratamiento suele extenderse tres veces más que en aquellos

Tabla 2. Clasificación de opioides

\begin{tabular}{|ll|}
\hline Opioides débiles & Opioides fuertes \\
\hline Tramadol* & Morfina* \\
\hline Codeína & Metadona* \\
\hline Dihidrocodeína & Oxicodona* \\
\hline Dextropropoxifeno & Fentanilo* \\
& Buprenorfina* \\
& Tapentadol* \\
& Hidromorfona \\
& Meperidina \\
\hline
\end{tabular}

*Opioides más relevantes en la práctica clínica. 
Figura 1. Flujograma de evaluación preoperatoria del paciente con dolor crónico no oncológico sometido a cirugía.

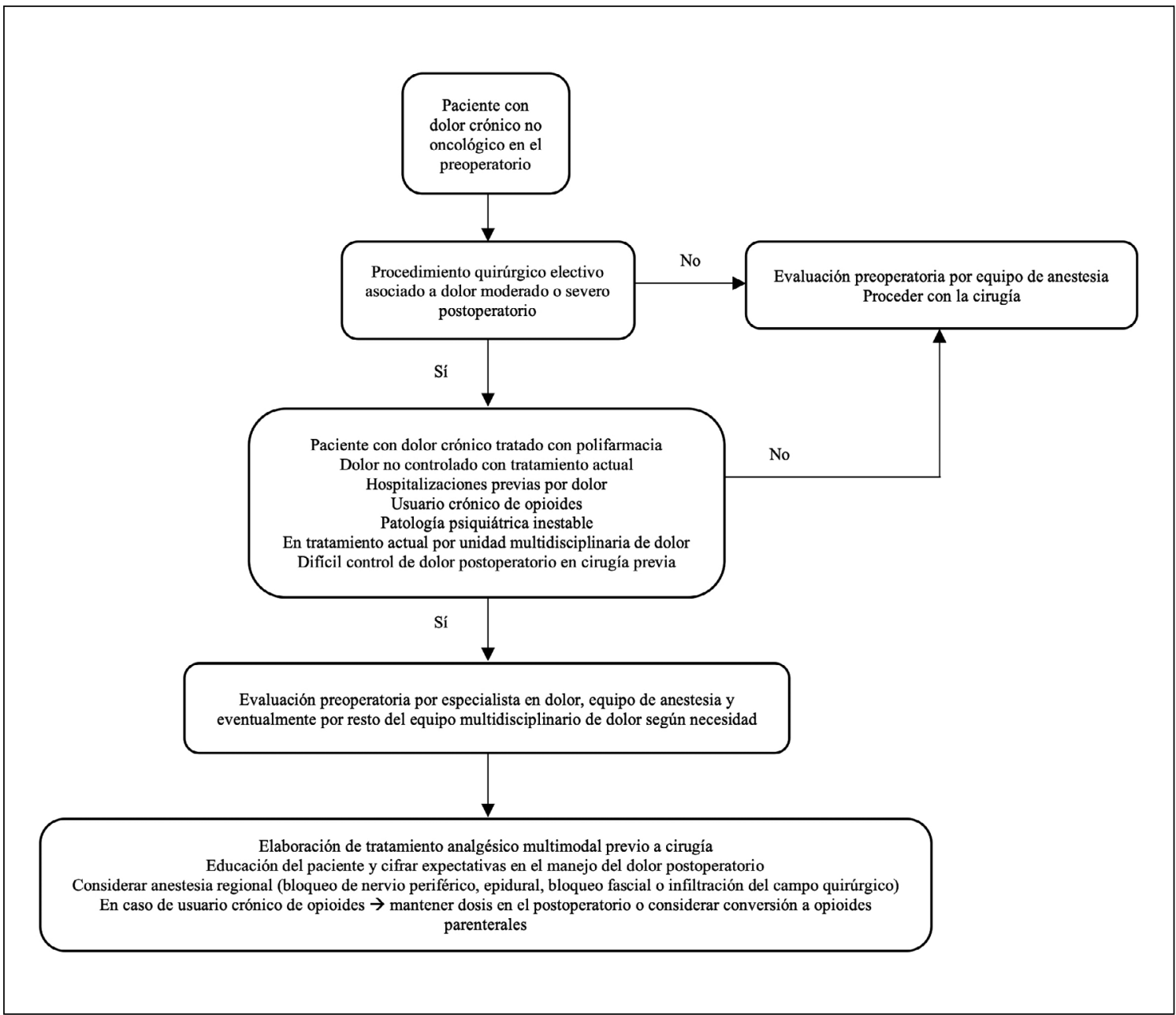

pacientes sin consumo crónico de opioides ${ }^{14}$. Para los pacientes que consumen opioides regularmente, estos deben mantenerse en el postoperatorio para evitar síntomas de abstinencia. Si el resto del esquema analgésico es efectivo, la dosis de estos podría reducirse en aproximadamente un $50 \%{ }^{8}$, más esto es logrado en la minoría de los casos. Los antagonistas de los opioides debieran ser evitados por el mismo motivo, y se debe estar atento a síntomas sugerentes del desarrollo de un síndrome de abstinencia (Tabla 3). Por el contrario, el aumento progresivo de las dosis de opioides está asociado a la aparición de efectos adversos importantes como constipación, íleo, depresión respiratoria entre otros. Es por esto que, en lo posible, se debe evitar una administración de dosis elevadas de opioides e idealmente administrar fármacos coadyuvantes o realizar una rotación de opioides, comenzando con un $50 \%$ de la dosis equivalente posterior a cada cambio ${ }^{4,15}$.
Tabla 3. Sintomatología asociada a abstinencia de opioides

Síntomas en pacientes con abstinencia de opioides

Náuseas y vómitos

Irritabilidad

Dilatación pupilar

Sudoración

Diarrea

Mialgias

Piloerección

Rinorrea

Lacrimación

Fiebre

Alteración ciclo sueño-vigilia 


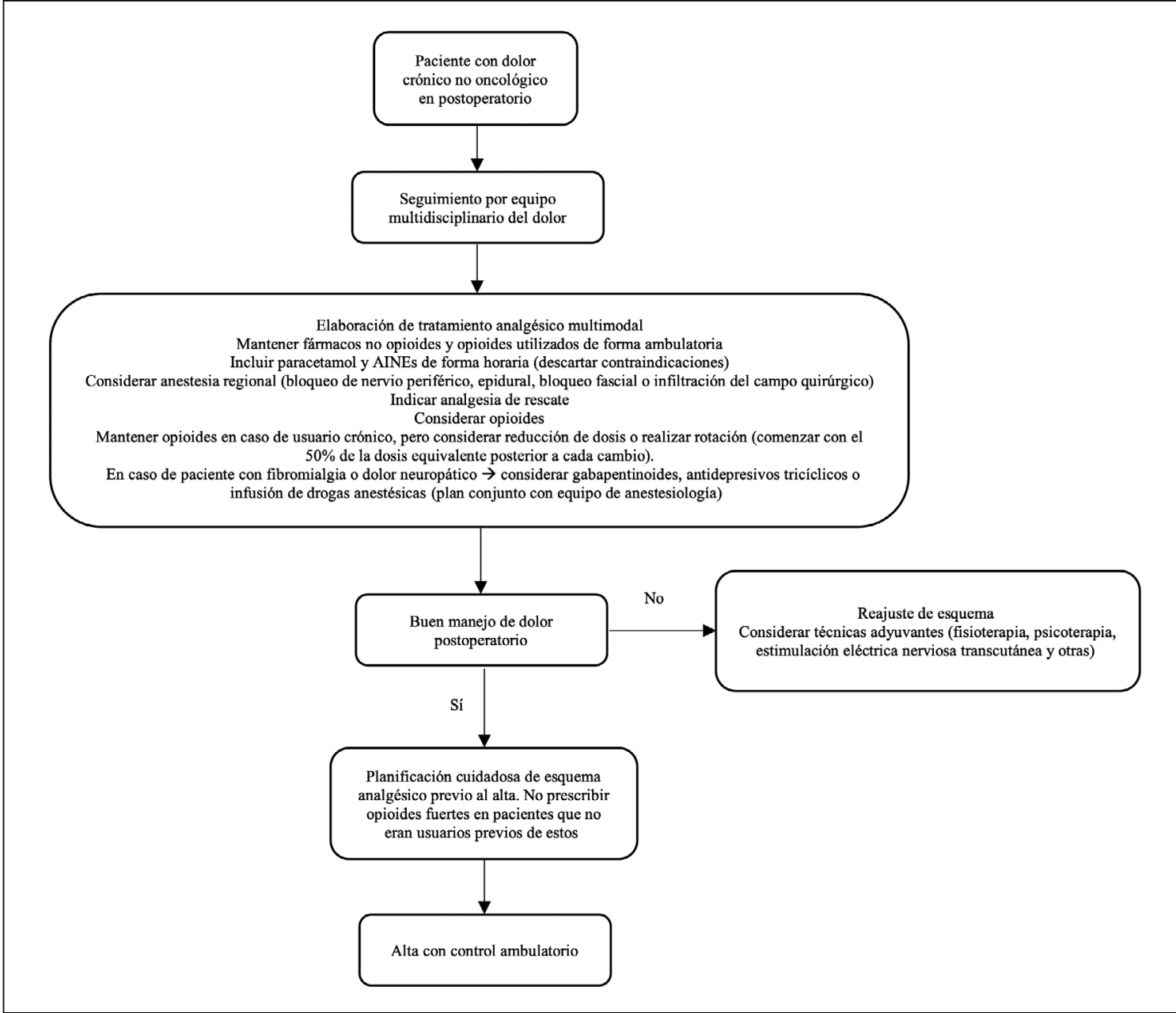

Como medida general, es relevante considerar el régimen alimentario de estos pacientes con el objetivo de evitar la constipación. Al comenzar la realimentación, esta debiese consistir en una dieta rica en fibras con indicación temprana de laxantes según metas.

Vale la pena destacar la particular importancia de la educación y la comunicación efectiva en este tipo de pacientes. Debe mantenerse un seguimiento, idealmente por un equipo multidisciplinario de dolor durante todo el período perioperatorio, fijando expectativas claras y tomando en consideración la sintomatología del paciente. De considerarlo necesario, existen otras técnicas adyuvantes que podrían proveer un mejor control del dolor y ansiedad como lo son la fisioterapia, psicoterapia, estimulación eléctrica nerviosa transcutánea entre otras $^{16}$.

Finalmente, debe realizarse una cuidadosa planificación con respecto al esquema analgésico previo al alta del paciente. En el contexto de la gran epidemia del uso problemático de opioides que se vive actualmente en Estados Unidos ${ }^{17}$, existen consideraciones particulares con respecto a la indicación de estos fármacos. En pacientes que no son usuarios de opioides fuertes, idealmente estos no debiesen ser prescritos luego del alta. Para aquellos que tienen indicación de uso de opioides, sus dosis, frecuencia y tiempo de administración debiesen ser la más baja dentro de lo posible. En caso contrario podríamos estar exponiendo al paciente al riesgo de convertirse en un usuario crónico y a una potencial adicción a estos fármacos en el futuro. Además, esta indicación debe ir claramente señalada en la epicrisis de los pacientes con una fecha de término del tratamiento y un seguimiento estrecho. Esto es con el objetivo de que exista un solo prescriptor de opioides por paciente, y evitar la duplicación de re-
Figura 2. Flujograma de manejo postoperatorio del paciente con dolor crónico no oncológico sometido a cirugía. 
cetas o consumo de grandes cantidades de opioides por estos pacientes.

\section{Conclusión}

El dolor crónico es una condición cada vez más prevalente en nuestra población. Este tiene distintas implicancias cuando un paciente con DCNO será sometido a una cirugía. El cuidado perioperatorio idealmente debe ser multidisciplinario, siendo fundamental una buena comunicación entre el equipo de cirugía y anestesiología. En general, los pacientes con DCNO tienen un umbral al dolor disminuido y requieren de una mayor cantidad de analgésicos. Además, estos pacientes muchas veces consumen o han intentado diversos tratamientos farmacológicos, complejizando o muchas veces limitando los esquemas analgésicos a utilizar. Debe considerarse, además, el aumento en el riesgo de distintas complicaciones que pueden añadir morbilidad al posto- peratorio. Existen distintos esquemas analgésicos en los cuales basarse, siendo muy relevante una adecuada planificación preoperatoria y un ajuste del tratamiento según las necesidades de cada paciente. Una comunicación efectiva entre el paciente y los distintos equipos involucrados en su cuidado, junto con un abordaje sistematizado del dolor, optimizará de esta manera su cuidado perioperatorio, mejorando su experiencia y resultados.

\section{Responsabilidades éticas}

Protección de personas y animales. Los autores declaran que para esta investigación no se han realizado experimentos en seres humanos ni en animales.

Confidencialidad de los datos. Los autores declaran que en este artículo no aparecen datos de pacientes.

Conflictos de interés: no hay.

\section{Bibliografia}

1. Bilbeny N, Miranda J, Eberhard M, Ahumada M, Méndez L, Orellana M, et al. Survey of chronic pain in ChilePrevalence and treatment, impact on mood, daily activities and quality of life. Scand J Pain. 2018;18:449-56.

2. Miclescu A. Chronic pain patient and anaesthesia. Rom J Anaesth Intensive Care. 2019;26:59-66.

3. Cozowicz C, Olson A, Poeran J, Mörwald E, Zubizarreta N, Girardi F, et al. Opioid prescription levels and postoperative outcomes in orthopedic surgery. Pain. 2017;158:2422-30

4. Coluzzi F, Bifulco F, Cuomo A, Dauri M, Leonardi C, Melotti R, et al. The challenge of perioperative pain management in opioid-tolerant patients. Ther Clin Risk Manag. 2017;13:1163-73.

5. Brown AK, Christo PJ, Wu CL. Strategies for postoperative pain management. Best Pract Res Clin Anaesthesiol. 2004;18:703-17.

6. Verret M, Lauzier F, Zarychanski R, Perron C, Savard X, Pinard A. Perioperative use of gabapentinoids for the management of postoperative acute pain. Anesthesiol. 2020;133:265-79.

7. Kumar A, Habib A. The role of gabapentinoids in acute and chronic pain after surgery. Curr Opin Anaesthesiol. 2019;32:629-34

8. Tumber P. Optimizing perioperative analgesia for the complex pain patient: medical and interventional strategies. Can J Anaesth. 2014;61:131-40.

9. Baldwin DS, Ajel K, Masdrakis VG, Nowak M, Rafiq R. Pregabalin for the treatment of generalized anxiety disorder: an update. Neuropsychiatr Dis Treat. 2013;9:883-92.

10. Mugabure Bujedo B, González Santos S, Tranque Bizueta I, Araujo López A, Torán García L. Manejo del dolor perioperatorio de los pacientes en tratamiento crónico con opioides. Rev Soc Esp Dolor 2009;16:288-97.

11. Dworkin RH, O'Connor AB, Audette J. Recommendations for the pharmacological management of neuropathic pain: an overview and literature update. Mayo Clin Proc. 2010; 85:3-14.

12. Pogatzki-Zahn EM, Englbrecht JS Schug SA. Acute pain management in patients with fibromyalgia and other diffuse chronic pain syndromes. Curr Opin Anaesthesiol. 2009;22:627-33.

13. Rapp SE, Ready LB, Nessly ML. Acute pain management in patients with prior opioid consumption: A case-controlled retrospective review. Pain. 1995;61:195201.

14. de Leon-Casasola OA, Myers DP, Donaparthi S, Bacon DR, Peppriell J, Rempel J, et al. A Comparison of Postoperative Epidural Analgesia Between Patients With Chronic Cancer Taking High Doses of Oral Opioids Versus Opioid-Naive Patients. Anesth Analg. 1993;76:302-7.

15. Lewis N, Williams J. Acute pain management in patients receiving opioids for chronic and cancer pain Contin Educ Anaesth Crit Care Pain. 2005;5:127-9.

16. Farrell C, McConaghy P. Perioperative management of patients taking treatment for chronic pain. BMJ 2012;345:e4148-e4148.

17. Vowles K, McEntee M, Julnes P, Frohe T, Ney J, van der Goes D. Rates of opioid misuse, abuse, and addiction in chronic pain. Pain. 2015;156:569-76. 